\title{
Numerical simulations of high frequency respiratory flows in a model bifurcating lung geometry
}

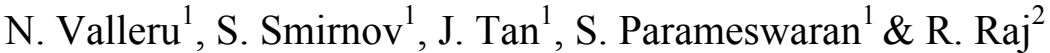 \\ ${ }^{I}$ Department of Mechanical Engineering, Texas Tech University, USA \\ ${ }^{2}$ Department of Internal Medicine, \\ Texas Tech University Health Sciences Center, USA
}

\begin{abstract}
Numerical studies of $2 \mathrm{D}$ cases are conducted using a CFD code FLUENT to analyze the flow patterns and gas transport at high oscillatory frequencies through a two-generation bifurcating lung model. The geometry corresponds to fifth to seventh generations of airways with the dimensions based on the Wiebel's symmetric pulmonary model. Computations are carried out for the Reynolds numbers $\operatorname{Re}=400$ and $\operatorname{Re}=1000$, while the Womersley number is Wo $=4.0$ and $\mathrm{Wo}=16.0$. The average mass distribution in the entire lung model is also investigated to analyze the influence of flow frequency on the mass diffusion efficiency. The numerical results of the current study pointed to: i) the numerical model successfully reproduces many results observed in the experiments; and ii) there is practically no net effect of the high frequency on the increased mass diffusion in the bifurcation geometry. The developed numerical model may be further used in more complicated 3D geometries and for determining the optimal conditions for artificial lung ventilation.
\end{abstract}

Keywords: pulmonary airways, oscillatory flow, diffusion, numerical simulation, CFD, fluid mechanics.

\section{Introduction}

To better understand the human pulmonary system, the knowledge of fluid mechanics is essential. When air passes through human lungs, it constantly changes its direction and speed, which leads to complex flow phenomena such as 
flow separation, recirculation, and mixing across airway cross-sections. Therefore, a physical description of the flow characteristics and gas transport in the pulmonary airways is essential. Although experimental studies can improve the insight, they are expensive and restricted in many ways due to the complex structure and small dimensions of the pulmonary airways. However, the rapid growth of the electronic computer technology enables us to utilize numerical simulation as a complement to the experimental studies. Moreover, once a numerical model is validated versus experimental measurements, it may be further used in studying the flow behavior in complex geometries where experimental studies are impossible.

\section{An experiment model}

The airways of human lungs are an extremely complex network of small bifurcations. This nature of the airways makes the study of flow and the transport of gases infeasible without reasonable simplifications. One of the famous and most commonly used simplified experimental models is provided by Wiebel and Gomez [1]. He conducted detailed measurements of human lungs and constructed a symmetric model by assuming the airways of any generation are identical and the surfaces are rigid, straight and smooth.

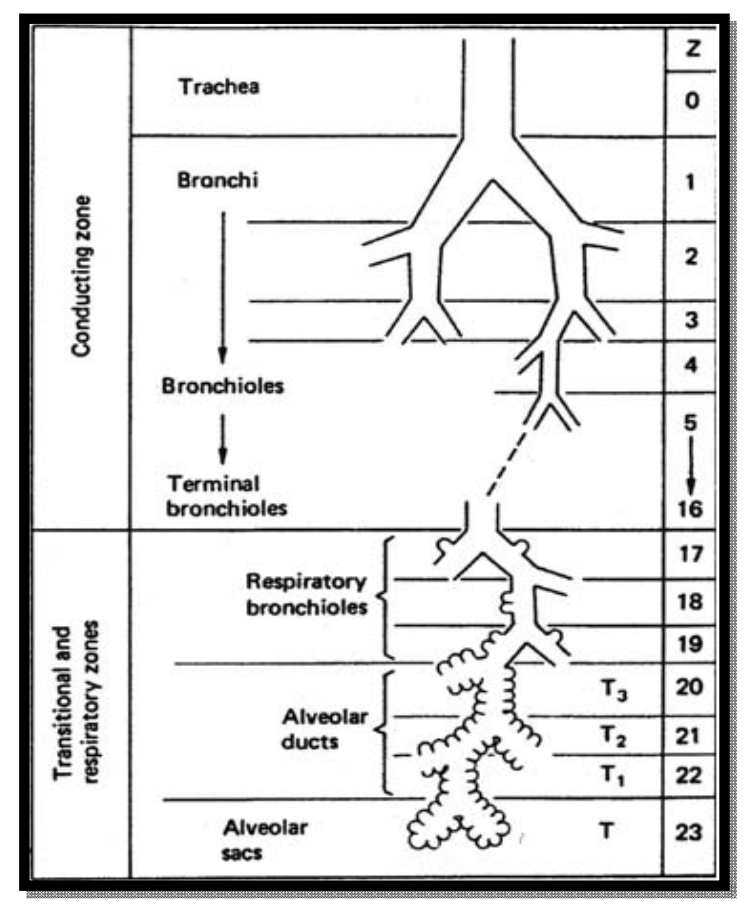

Figure 1: Wiebel's model. 
Table 1: $\quad$ Parameters based on Wiebel's model.

\begin{tabular}{|c|c|c|c|c|}
\hline $\begin{array}{c}\text { Generation } \\
\underline{n} \\
\end{array}$ & $\frac{D}{(\mathrm{~mm})}$ & $\begin{array}{c}\underline{L} \\
(\mathrm{~mm})\end{array}$ & $\underline{\mathrm{Re}}$ & $\underline{\alpha}$ \\
\hline 5 & 3.50 & 10.7 & 341.6 & 0.54 \\
\hline 6 & 2.80 & 9.0 & 213.5 & 0.43 \\
\hline 7 & 2.30 & 7.6 & 130.0 & 0.35 \\
\hline
\end{tabular}

The process of breathing can be split into two phases: the inspiratory phase and the expiratory phase. Because of the oscillatory nature, the lung flow behaves in an unsteady manner and thus cannot be characterized by the Reynolds number alone. Instead, the Womersley number is widely accepted to describe the oscillatory flow. It is defined as:

$$
W o=\frac{D}{2} \sqrt{\frac{\omega \rho}{\mu}}
$$

where $W_{O}=$ the Womersley number, $D=$ the airway diameter, $\omega=$ the frequency of oscillation, $\mu=$ the dynamic viscosity.

In the experiment, steady-state Taylor-type dispersion is conducted as shown in Figure 2. At the beginning, the straight tube is filled with fluid A. When $t=t_{o}$, another fluid B is introduced into the tube at the inlet boundary with a parabolic velocity profile. After a certain period of time, when $t=t_{1}$, the dispersion of fluid $\mathrm{B}$ is clear enough to be observed and measured. The apparent diffusion coefficient is hereby introduced to quantify the diffusion and it is defined as:

$$
D_{a p p}=\frac{\alpha^{2} U^{2}}{48 D_{m o l}}
$$

where $D_{m o l}=$ the molecular diffusion coefficient, $\alpha=$ the radius of tube, $U=$ the average velocity of fluid $\mathrm{B}$ at the inlet boundary.

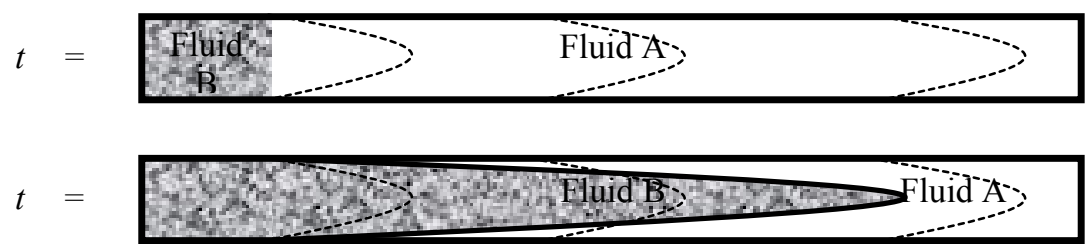

Figure 2: Taylor's dispersion. 


\section{Numerical techniques}

In numerical simulations we modeled a double bifurcation ( $5^{\text {th }}$ to $7^{\text {th }}$ generation) of the lung airway network. Finite volume method was utilized to discretize the general transportation equation:

$$
\frac{\partial(\rho \phi)}{\partial t}+\nabla \cdot(\rho \vec{U} \phi)=\nabla \cdot(\mu \nabla \phi)+S
$$

The second order UPWIND scheme and SIMPLE algorithm were performed to solve for the velocity and pressure distributions. All calculations were processed with double precision to ensure the accuracy of the results.

GAMBIT software package was utilized to model and mesh the airways' geometry, and FLUENT software package was used to solve the fluid governing equations to obtain the data on the flow field and fluid dispersion.

The mesh generation for the straight tube is straight forward. Quadrilateral mesh was generated for the whole fluid domain, and the mesh density was increased near the tube wall surface due to the boundary layer effects.

For the double bifurcation model, the whole fluid domain was divided into different sections to accommodate the requirements of different flow regions (Figure 3). Quadrilateral mesh is applied for the straight tube sections and triangular mesh is generated to fit the geometry of the junction area. Also mesh density is increased near the surfaces of the wall boundaries to take care of the boundary layer effects.

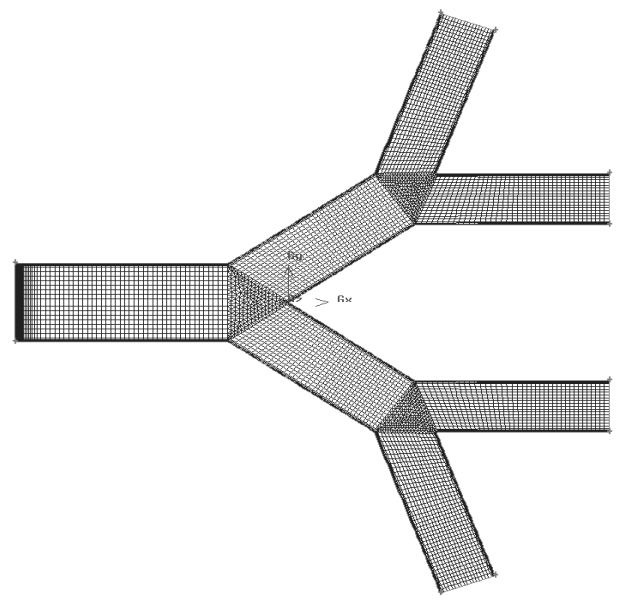

Figure 3: $\quad$ Mesh for the double bifurcation model.

Two different numerical models were constructed and analyzed in the current study. Namely, 1) 2D double bifurcation scaled up model, and 2) 2D double bifurcation actual size model. For each model, we conduct research on both 
steady-state and unsteady cases, change the inlet flow frequency and compare the results in order to uncover how the oscillatory frequency affects the flow diffusion.

Generally the inlet velocity profile is specified as:

$$
u=u_{0} \sin (\omega t), \text { where } u_{0}=\frac{\partial p}{\partial x} \frac{r^{2}-a^{2}}{4 \mu}
$$

$u=$ the axial velocity for unsteady case, $u_{0}=$ the axial velocity for steady case, $\partial p / \partial x=$ the axial pressure gradient, $a=$ the radius of tube, $r=$ the radial position, $\mu=$ the dynamic viscosity, $t=$ the time and $\omega=$ the oscillatory frequency.

The transport equation is solved using the following boundary conditions: (i) at the inlet boundary, the mass fraction of fluid $\mathrm{B}$ is fixed to 1.0 and the mass fraction of fluid $\mathrm{A}$ is fixed to 0.0 , (ii) at the outlet boundary, the mass fraction of fluid $\mathrm{B}$ is fixed to 0.0 and the mass fraction of fluid $\mathrm{B}$ is fixed to 1.0. Moreover, for all unsteady cases, the initial conditions are always the same: at $t=0$, the whole fluid domain is filled with fluid A, which means that the mass fraction of fluid $\mathrm{B}$ is equal to zero at $t=0$ everywhere in the fluid domain, except at the inlet boundary.

\section{Simulation results and discussions}

\subsection{D scaled-up double bifurcation model}

The simulations were carried out for the inspiratory flow with the Reynolds number $\mathrm{Re}=410$ and the average velocity of $0.00137 \mathrm{~m} / \mathrm{s}$. Water (at the room temperature) was used as the working medium.

We calculated the average non-dimensional velocities at different cross sections along the model (such as cross section 2B, 3B 2C, 3C, see Figure 4 below), and compared these data with the experimental results from literature [2]. In Figure 4, it is clearly indicated that numerical and experimental results reach a satisfactory agreement, which provides a solid validation for our numerical models.

\subsection{D double bifurcation model with actual size}

Since we have enough confidence in the 2D scaled-up double-bifurcation model, the next step was to apply it to the actual size model of a very small scale.

Simulations are carried out for two cases with different Reynolds numbers $\mathrm{Re}=400$ and $\mathrm{Re}=1000$.

The validation of this actual size model is performed by comparing the flow velocity distribution between the numerical results from simulations and the numerical results from literature [3]. As shown in Figure 5, the numerical results are well matched. 

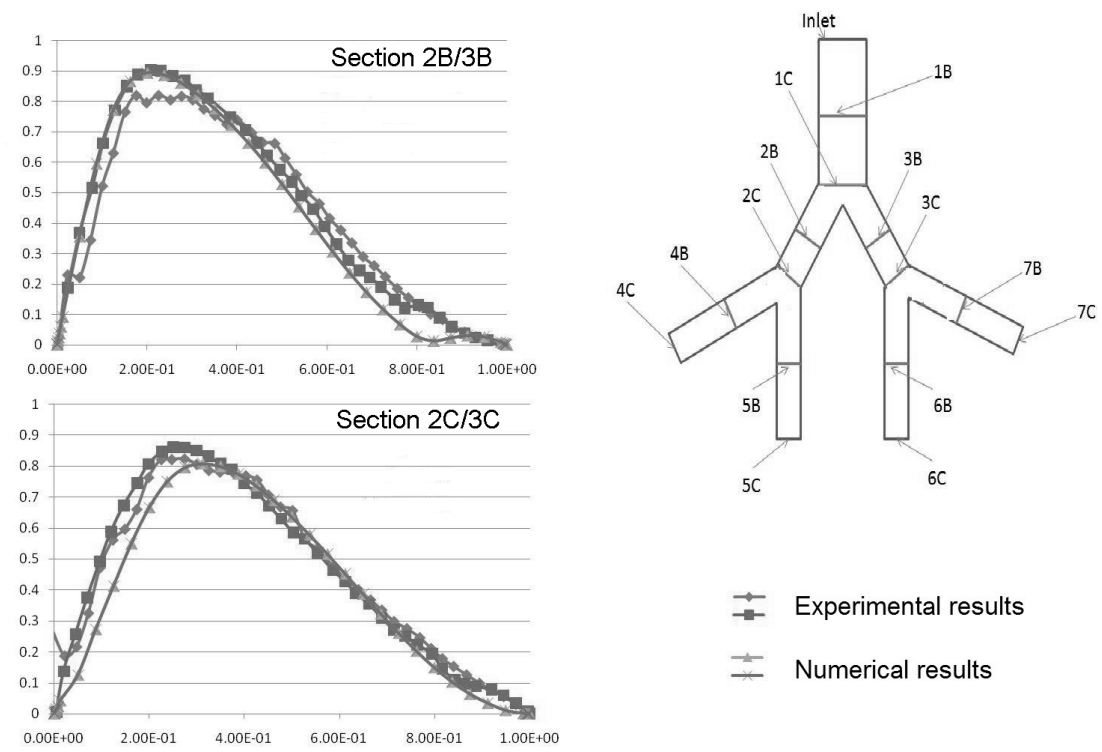

Figure 4: Scaled-up model, non-dimensional velocity (vertical axis) vs. reduced distance (horizontal axis), the numerical results compared with experimental results.
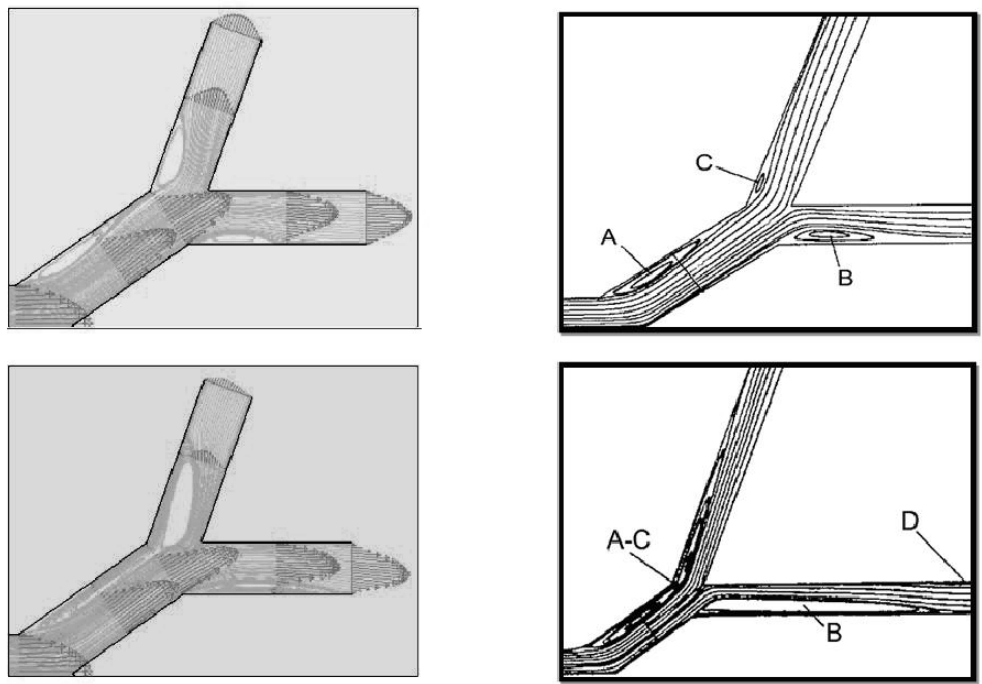

Figure 5: Actual size model, velocity distribution comparison, numerical results vs. results from literature [3], $\mathrm{Re}=400$ and $\mathrm{Re}=1000$. 
After validating our model, we studied two cases with the same Reynolds number $R e=1000$, but different Womersley numbers, Wo $=4.0$ and $\mathrm{Wo}_{0}=16.0$. Different Womersley numbers correspond to different flow frequencies. We were curious about whether the inlet flow frequency has any effect on the fluid diffusion or not. In other words, the purpose of this simulation was to study whether increasing the inlet flow frequency causes an increase of the mass fraction of fluid B at all four outlets of our model.

The results of simulation have to be compared at exactly the same phases of oscillation due to the periodic nature of the flow at inlet boundary. The results also have to be compared at the same absolute flow times, because the molecular diffusion depends on the absolute time. So we had to follow two criteria: 1) The results are chosen at the same absolute flow time, and 2) they are chosen at the same phase of a cycle, e.g., peak of the inspiratory phase, peak of the expiratory phase, etc.

In this simulation, we compute both cases under exactly the same boundary conditions and numerical set-ups (e.g., grid size, time step, etc.). Furthermore, the absolute time is chosen to be large enough (five cycles for Wo $=4$ ) to guarantee that the periodic flow becomes well established inside the bifurcation model. The results are compared at the same phase of the last cycle for both cases.

Tables 2 and 3 present the results of such computations. The mass fraction of oxygen (fluid B) is computed at four outlet boundaries. The flow time is 0.4009 seconds, which equals to five cycles for $\mathrm{Wo}=4.0$ case, and eighty cycles for $\mathrm{Wo}_{\mathrm{O}}=16.0$ case.

\section{Conclusion}

The data in Tables 2 and 3 reveal the fact that at the end of the last cycle, the mass fraction is essentially zero for both values of the Womersley number. At the peak of the inspiratory phase the values of mass fraction of oxygen at the outlets are much larger for $\mathrm{Wo}_{\mathrm{O}}=4$ than for $\mathrm{Wo}_{\mathrm{O}}=16$. Based on this fact, our conclusion is that there is practically no net effect of the high frequency (or Womersley number) on the improved mass transfer in the bifurcation model.

Table 2: Mass fraction of oxygen at four outlet boundaries at the peak of the inspiratory phase (flow time $0.4009 \mathrm{~s}$ ).

\begin{tabular}{|c|c|c|}
\hline & Wo=4.0 & Wo=16.0 \\
\hline $\mathbf{1}^{\text {st }}$ outlet (7C) & 0.330531 & 0.003020 \\
\hline $\mathbf{2}^{\text {nd }}$ outlet (6C) & 0.529589 & 0.002776 \\
\hline $\mathbf{3}^{\text {rd }}$ outlet (5C) & 0.529775 & 0.002781 \\
\hline $\mathbf{4}^{\text {th }}$ outlet (4C) & 0.330554 & 0.003019 \\
\hline
\end{tabular}


Table 3: Mass fraction of oxygen at the outlet boundaries at the end of the cycle (flow time 0.4009s).

\begin{tabular}{|c|c|c|}
\hline & Wo=4.0 & Wo=16.0 \\
\hline $\mathbf{1}^{\text {st }}$ outlet (7C) & $6.917803 \mathrm{E}-19$ & $3.058330 \mathrm{E}-5$ \\
\hline $\mathbf{2}^{\text {nd }}$ outlet (6C) & $6.801282 \mathrm{E}-16$ & $2.807442 \mathrm{E}-5$ \\
\hline $\mathbf{3}^{\text {rd }}$ outlet (5C) & $6.972378 \mathrm{E}-16$ & $2.813371 \mathrm{E}-5$ \\
\hline $\mathbf{4}^{\text {th }}$ outlet (4C) & $7.056232 \mathrm{E}-19$ & $3.057900 \mathrm{E}-5$ \\
\hline
\end{tabular}

In other words, increasing oscillatory frequency does not cause any significant increase in the oxygen diffusion. We hypothesize that the situation might be different in 3D geometry, which opens the possibility for transverse flow instabilities, and as a result increased mixing and mass transfer along the bifurcation model. We intend to explore this possibility in the near future.

\section{References}

[1] Wiebel, E.R. and Gomez, D.M., Architecture of Human Lung, Science, Volume 137 No. 3530, pp. 577-585, 1962.

[2] Theunissen, R. and Riethmuller, M.L., Particle Image Velocimetry in Lung Bifurcation Models, Springer Berlin/Heidelberg, Volume 112, 2008 .

[3] Wilquem, F. and Degrez, G., Numerical Modeling of Steady Inspiratory Airflow Through a Three-Generation Model of the Human Central Airways, Journal of Biomechanical Engineering, Volume 119 Issue 1, pp. 59-66, 1997

[4] Chang, H.K., Mechanisms of gas transport during ventilation by highfrequency oscillation, Journal of Applied Physiology, Volume 56, Issue 3, pp. 553-563, 1984

[5] Pedley, T.J., Pulmonary Fluid Dynamics, Annual Review of Fluid Mechanics, Volume 9, pp. 229-274, 1977 